\title{
BMJ Open A minimally invasive technique for decompression of Chiari malformation type I (DECMI study): study protocol for a randomised controlled trial
}

\author{
Yu Hu, Jiagang Liu, Haifeng Chen, Shu Jiang, Qiang Li, Yuan Fang, Shuhui Gong, \\ Yuelong Wang, Siqing Huang
}

To cite: $\mathrm{Hu}$ Y, Liu J, Chen $\mathrm{H}$, et al. A minimally invasive technique for decompression of Chiari malformation type I (DECMI study): study protocol for a randomised controlled trial. BMJ Open 2015;5:e007869. doi:10.1136/bmjopen-2015007869

- Prepublication history for this paper is available online. To view these files please visit the journal online (http://dx.doi.org/10.1136/ bmjopen-2015-007869).

$\mathrm{YH}$ and $\mathrm{JL}$ contributed equally.

Received 4 February 2015 Revised 31 March 2015 Accepted 9 April 2015

CrossMark

Department of Neurosurgery, West China Hospital, Sichuan University, Chengdu, Sichuan Province, China

Correspondence to Dr Siqing Huang; huangsiqing028@163.com

\section{ABSTRACT}

Introduction: Chiari malformation type I (CM-I) is a congenital hindbrain anomaly that requires surgical decompression in symptomatic patients. Posterior fossa decompression with duraplasty (PFDD) has been widely practiced in Chiari decompression, but dural opening carries a high risk of surgical complications. A minimally invasive technique, dural splitting decompression (DSD), preserves the inner layer of the dura without dural opening and duraplasty, potentially reducing surgical complications, length of operative time and hospital stay, and cost. If DSD is non-inferior to PFDD in terms of clinical improvement, DSD could be an alternative treatment modality for CM-I. So far, no randomised study of surgical treatment of CM-I has been reported. This study aims to evaluate if DSD is an effective, safe and cost-saving treatment modality for adult CM-I patients, and may provide evidence for using the minimally invasive procedure extensively. Methods and analysis: DECMI is a randomised controlled, single-masked, non-inferiority, single centre clinical trial. Participants meeting the criteria will be randomised to the DSD group and the PFDD group in a 1:1 ratio. The primary outcome is the rate of clinical improvement, which is defined as the complete resolution or partial improvement of the presenting symptoms/signs. The secondary outcomes consist of the incidence of syrinx reduction, postoperative morbidity rates, reoperation rate, quality of life (QoL) and healthcare resource utilisation. A total of 160 patients will be included and followed up at 3 and 12 months postoperatively.

Ethics and dissemination: The study protocol was approved by the Biological and Medical Ethics Committee of West China Hospital. The findings of this trial will be published in a peer-reviewed scientific journal and presented at scientific conferences.

Trial registration number: ChiCTR-TRC-14004099.

\section{BACKGROUND}

Chiari malformation type I (CM-I), originally described by Hans Chiari in 1891, is a congenital hindbrain deformity that is characterised by caudal tonsillar herniation

\section{Strengths and limitations of this study}

- This study is the first randomised control trial for Chiari malformation type I (CM-I)

- This trial will provide the neurosurgical community with robust evidence of whether a minimally invasive technique is the best option in Chiari decompression.

- Healthcare resource utilisation will also be evaluated.

- Our centre is one of the largest neurosurgical centres in China and covers nearly $8 \%$ of the entire Chinese patient population.

- This trial is a single centre randomised controlled study.

through the foramen magnum and a small posterior fossa, leading to neural structure compression and a cerebrospinal fluid (CSF) flow disturbance at the craniocervical junction (CVJ). ${ }^{1-3}$ To decompress the neuraxis and restore the normal CSF dynamics, surgical intervention is indicated in symptomatic patients. Suboccipital craniectomy and cervical laminectomy are broadly used and accepted as the mainstay of surgical treatment of CM-I. ${ }^{4}$ However, surgical adjuvants after bone decompression, such as dural opening and duraplasty, remain subject to considerable controversy because of the inconsistent outcomes and side effects among those procedures. ${ }^{25-7}$

Posterior fossa decompression with duraplasty (PFDD), involving bone decompression, dural opening and a variety of intradural procedures followed by duraplasty, has been widely used and proved effective in Chiari

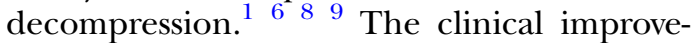
ment rate of patients undergoing PFDD was $78.6 \%(44 / 56)$ in a meta-analysis. ${ }^{4}$ A comparable rate of clinical response $(82.2 \%)$ was also reported by Hoffman et $a l^{1}$ through a 
literature review, in which 231 patients were included. Although PFDD is an effective surgical technique for CM-I patients, surgical complications, such as CSF leakage, meningitis and pseudomeningocoele, which may result in considerable suffering and resource consumption, have frequently been reported. ${ }^{14} 710$ The average incidence of complications was approximately $24.4 \%(33 / 135)$ in the meta-analysis and $12.4 \%$ (28/226) in the literature review. ${ }^{14}$

To reduce surgical morbidity, dural splitting decompression (DSD), an extradural procedure, was introduced by Isu et $a l^{11}$ in 1993. Only superficial wound infection has been documented in the literature in patients undergoing DSD. ${ }^{5} 79^{11-17}$ Moreover, DSD preserves the inner layer of the dura without duraplasty, and thus it is associated with less surgical time, shorter length of hospital stay and lower cost of healthcare, when compared with widely used PFDD. ${ }^{4}{ }^{10}$ As to the efficacy of DSD, multiple studies have reported a comparable clinical response rate that ranged from $72.2 \%$ to $100.0 \%$ in CM-I with or without syringomyelia. ${ }^{6} 911$ 13-18 The comparable efficacy, fewer complications, shorter length of operative time and hospital stay, and lower costs, in comparison with the widely practiced PFDD, should prompt the neurosurgical community to consider whether DSD could be used as an alternative treatment of PFDD in CM-I patients. ${ }^{4} 710$

The current literature fails to provide the surgical community with robust evidence (class I and class IIa studies) of which surgical procedure is the best option in the treatment of CM-I. ${ }^{4}{ }^{19}$ DECMI is designed to evaluate which treatment modality is the best option, with the primary aim being to demonstrate that DSD is non-inferior to PFDD in terms of clinical improvement.

\section{STUDY OBJECTIVE}

In this study, we aim to test the hypotheses that DSD is not inferior to PFDD in terms of clinical improvement in the treatment of adult CM-I patients with or without syringomyelia, and that DSD has additional benefits over PFDD, including fewer complications, shorter length of operative time and hospital stay, better quality of life (QoL) and lower cost.

\section{METHODS}

\section{Study design}

This trial is a single centre, single-masked, randomised controlled study with two parallel comparison groups. The overall flow of the protocol is presented in figure 1. The study protocol was performed at West China hospital, which is one of the largest neurosurgical centres in China. All Chiari decompression will be performed by the senior author $(\mathrm{SH})$.

\section{Study population}

All patients presenting with CM-I will be assessed for eligibility with the inclusion and exclusion criteria detailed

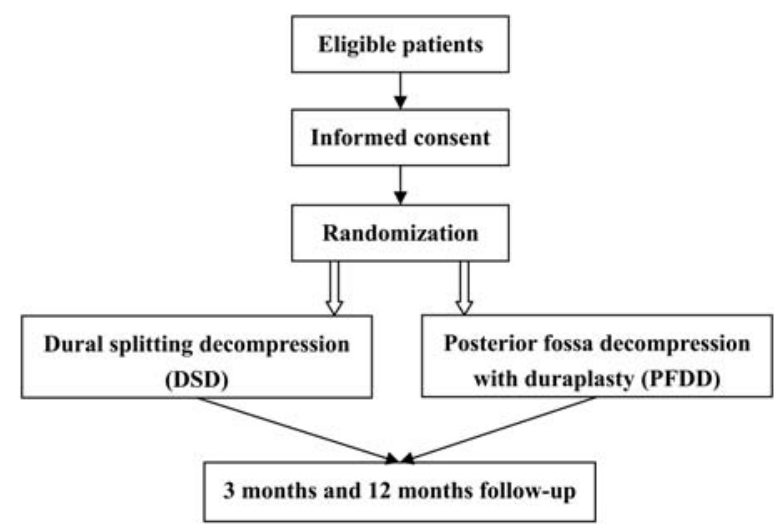

Figure 1 Decompression of Chiari malformation type I (DECMI) trial flow chart.

in table 1. Eligible patients will be stratified by the presence of syringomyelia, and then randomised to undergo either DSD or PFDD.

\section{Sample size}

We began performing DSD in CM-I patients with or without syringomyelia starting in June 2013, and therefore 1 year outcome data of those patients is lacking. We found that the rate of clinical improvement in adult CM-I patients undergoing DSD ranges from $75 \%$ to $100 \%$ (average 87\%) according to the literature. $^{6} 911121618$ Based on the preliminary data of our department, the rate of clinical response was nearly $82 \%$ in patients treated with PFDD (unpublished). Thus, a sample size of 142 patients (71 in each group) would be required with a $5 \%$ (two-sided) type I error and a power of $80 \%$ using a $10 \%$ non-inferiority margin. In consideration of those lost to follow-up, 80 patients in each group will be recruited for this trial.

\section{Randomisation and blinding}

Through a minimisation software, the eligible patients will be allocated using the minimisation method with a preset variable-the presence of syringomyelia. After

Table 1 Inclusion and exclusion criteria for trial participants

\begin{tabular}{|c|c|}
\hline Inclusion criteria & Exclusion criteria \\
\hline $\begin{array}{l}\text { I. Age between } 18 \\
\text { and } 60 \text { years } \\
\text { II. Symptomatic CM-I } \\
\text { III. Informed consent }\end{array}$ & $\begin{array}{l}\text { I. Dysfunction of blood coagulation } \\
\text { II. Pregnancy } \\
\text { III. History of meningitis, PFD or } \\
\text { shunt surgery } \\
\text { IV. Hydrocephalus, craniospinal } \\
\text { instability, severe ventral } \\
\text { compression in CVJ, spinal cord } \\
\text { tumour, tethered spinal cord, } \\
\text { arachnoid cyst of the posterior } \\
\text { fossa }\end{array}$ \\
\hline
\end{tabular}


patient information is imported, participants will be randomised to the DSD group or the PFDD group. A special trialist who is masked to the study participants will perform the randomisation process. Sealed envelopes containing treatment assignments will be used to conceal allocation until just before the operation. In the single-masked trial, the patients will be blinded to the assigned treatment. The surgeons, however, will not be masked because of performing the surgery. To minimise measurement bias, two investigators who are blinded to the treatment information will collect outcome data. The statistician will not participate in the implement of the study, and will be blinded to the trial intervention.

\section{Treatment protocol}

All symptomatic patients suspected for CM-I will undergo craniocervical MRI to confirm the diagnosis. If diagnosed with CM-I, patients will further have a head CT scan performed to exclude hydrocephalus, threedimensional CT to exclude atlantoaxial instability and/ or severe basilar invagination, and whole-spine MRI to exclude spinal tumours and tethered cord syndrome, and to determine the extension of syrinx.

When informed consent is obtained from eligible patients, randomisation will take place. The allocated surgical treatment will be performed as soon as possible after randomisation.

\section{Group A: Dural splitting decompression}

Surgery in the left lateral position will be performed under general anaesthesia. A midline skin incision will be made extending from the external occipital protuberance to the upper cervical spine. The muscular attachments at the superior nuchal line and the C2 (if C2 laminectomy will not be required) will be left intact. A small suboccipital craniectomy $(3 \mathrm{~cm}$ in diameter) and a laminectomy of $\mathrm{C} 1(2.5 \mathrm{~cm}$ in diameter $)$ will be performed uniformly with the help of a high-speed surgical drill and rongeurs in all patients. Additional cervical laminectomy will be performed depending on the extent of cerebellar herniation. After osseous decompression, any craniocervical epidural adhesion band will be coagulated and carefully removed. Once the cervico-occipital dura is well exposed, a vertical incision of the outer layer will be performed, and the outer layer of the dura will be separated and removed from the inner layer caudally and laterally under microscopic visualisation. To avoid shrinking the inner leaf of the dura, Gelfoam rather than bipolar cautery will be used to control any venous haemorrhage. If accidental opening of the inner layer of the dura occurs, a stitch will immediately be performed with suture (5-0 VICRYL, Johnson \& Johnson). The muscles will be closed without any tension, and the fascia and subcutaneous plans will be tightly closed.

\section{Group B: Posterior fossa decompression with duraplasty}

Prior to dural opening, the procedures in patients undergoing PFDD will be similar to the procedures in those undergoing DSD. Then the dura will be opened in a Y-shaped incision under microscopic visualisation, and care will be taken to leave the arachnoid intact. The grade of arachnoid pathology as described elsewhere (grade $0=$ no detectable arachnoid pathology; grade $1=$ slight arachnoid adhesions, arachnoid translucent; Grade 2=severe arachnoid adhesions, arachnoid not translucent), and accidental opening of the arachnoid membrane, will be recorded in the $\mathrm{CRF}^{2}$ Severe arachnoid adhesions will be resected, and dural patch grafting will be performed with rhombic shape $2.5 \times 2.5$ artificial dura (DURA-Guard, Johnson \& Johnson) in a tight running fashion to expand the posterior fossa and reconstruct a spacious cistern magna. The wound will then be closed in layers.

\section{Follow-up}

Patients will be followed up at 3 and 12 months after surgical decompression.

\section{Outcomes}

To ensure the accuracy and reproducibility of the outcome, two investigators who have already participated in a 2-day meeting, focusing on the rationale, surgical procedures and data collection and management of the trial, will perform the outcome assessment.

The primary outcome of the trial will be the rate of clinical improvement, which is defined as the complete resolution or partial improvement of the presenting symptoms/signs. The secondary outcomes of the study will include: (1) the incidence of syrinx reduction: defined as a reduction of the syrinx cavity size; (2) postoperative morbidity rates: defined as CSF leakage, meningitis (bacterial or aseptic), pseudomeningocoele, postoperative hydrocephalus, subdural hygroma, bleeding complications and wound infection; (3) the reoperation rates: defined as a revision surgery for progression of symptoms/signs or complications that need surgical intervention; (4) QoL: which will be assessed by SF-12 Physical Component Scale (SF-12 PCS) and the Chiari Symptom Profile (CSP) as described elsewhere; ${ }^{320}$ and (5) the healthcare resource utilisation: defined as the length of operative time and hospital stay, and the total cost for the primary hospitalisation.

\section{Data collection and management}

Each participant will have a CRF with an anonymous study number, which will be used to record all protocolrequired information. The CRF is divided into the treatment forms and the follow-up forms, and is printed on two different papers. The schedule for the study visits and data collection is summarised in table 2 . On admission, baseline characteristics (ie, age, gender and the presence of syringomyelia) of eligible patient will be documented by the study investigator and sent to the special trialist for randomisation. The neurosurgeon will fill out the treatment forms, including the detailed surgical procedures and intraoperative findings, the 
Table 2 Schedule of the assessments

\begin{tabular}{|c|c|c|c|c|c|}
\hline Time point & $\begin{array}{l}\text { Enrolment } \\
\text {-1 day }\end{array}$ & $\begin{array}{l}\text { Allocation } \\
0 \text { day }\end{array}$ & $\begin{array}{l}\text { Intervention } \\
1 \text { days }\end{array}$ & $\begin{array}{l}\text { Follow-up } \\
\text { After } 3 \text { months }\end{array}$ & After 12 months \\
\hline \multicolumn{6}{|l|}{ Enrolment } \\
\hline Eligibility screen & $x$ & & & & \\
\hline Informed consent & $x$ & & & & \\
\hline Allocation & & $x$ & & & \\
\hline \multicolumn{6}{|l|}{ Interventions } \\
\hline DSD & & & $x$ & & \\
\hline PFDD & & & $\mathrm{X}$ & & \\
\hline \multicolumn{6}{|l|}{ Assessments } \\
\hline Demographics & $x$ & $x$ & & & \\
\hline Symptoms/signs & $x$ & $x$ & & $x$ & $x$ \\
\hline Syrinx & $x$ & $x$ & & $x$ & $x$ \\
\hline Surgical morbidity & & & $x$ & $x$ & $x$ \\
\hline Re-operation & & & & $x$ & $x$ \\
\hline SF-12 PCS & & $x$ & & $x$ & $x$ \\
\hline CSP & & $x$ & & $x$ & $x$ \\
\hline Resource utilisation & & & $\mathrm{X}$ & $x$ & $x$ \\
\hline
\end{tabular}

complications during hospitalisation, laboratory and radiological data and hospitalisation costs. After discharge, two trained outcome assessors will complete the follow-up forms by phone or in the outpatient clinic. After the information of participants is collected, the CRF should be completed as soon as possible. The investigators should give an explanation for any missing data. The completed CRF will be reviewed by an authorised investigator. A double-entry method will be used for abstracting data from the CRF. All entered data will be locked in a password-secure desktop computer, and will be checked by the principal investigator every month. At the end of the study, the database will be sent to the statistician for statistical analysis. All CRFs will be retained for up to 5 years by the principal investigator.

\section{Confidentiality}

To assure confidentiality, trial participants will be allocated an anonymous study number throughout the trial.

\section{Adverse and severe adverse events (AEs)}

All participants in the trial will be monitored for adverse events (AEs), which are defined as undesirable incidents occurring in patients during the study. All end point relevant complications (eg, CSF leakage, meningitis and pseudomeningocoele), however, will be recorded on the CRF not as AEs but as end points. A serious AE (SAE) is defined as death, life-threatening events, or significant disability. All AEs and SAEs will be documented on CRF in detail, and will be reported to the Quality Monitoring Board (QMB) and Biological and Medical Ethics Committee (BMEC). The principal investigator should report the SAE within $24 \mathrm{~h}$ after the SAE becomes known. Participants who suffer harm will be given medical treatment as compensation.

\section{Ethics and dissemination}

The trial will be carried out in accordance with the declaration of Helsinki and in the context of good clinical practice. Patients will be fully informed about the trial as well as given written informed consent prior to randomisation. The trial was registered in the Chinese Clinical Trial Registry (ChiCTR-TRC-14004099, 10 January 2014). Recruitment of participants started in June 2014. The duration of the trial is expected to be 3 years.

The findings of this trial will be presented at one or more major scientific conferences and published in a peer-reviewed scientific journal.

\section{Statistical analysis}

The intention-to-treat analysis will be performed in all participants, and patients without major protocol violations will have a per protocol analysis. The following baseline characteristics, including age, sex, the presenting symptoms/signs, the duration of symptoms/signs and the presence of syringomyelia, will be described and assessed for any imbalance between the two surgical groups. An imbalance will be considered if any of those characteristics between the two groups have a p value $<0.05$.

The continuous data will be analysed by independent sample $\mathrm{t}$ test or one-way analysis of variance. $\chi^{2}$ Test or Fisher's exact test will be used for nominal data. The effect of multivariables for clinical improvement will be analysed by logistic regression. ORs will be reported with a corresponding $95 \%$ CI. A p value $<0.05$ in two-tailed test is considered statistically significant.

After $1 \frac{1}{2}$ years of recruitment, interim analysis will be performed to compare the primary end point between the two surgical groups. 


\section{DISCUSSION}

Surgical decompression is the only treatment available for symptomatic CM-I patients, particularly those with progressive symptoms/signs. ${ }^{2} 313$ The main goals of surgical decompression are to: (1) decompress the neural structures; (2) restore the normal CSF dynamics at the CVJ; and (3) reduce the surgical morbidity and mortality. ${ }^{5} 717$ Therefore, the safest and most effective surgical procedure is advocated. ${ }^{7}{ }^{19}$ Currently, posterior fossa decompression without duraplasty, leaving the dura intact, is considered to be the safest surgical procedure, but the procedure is associated with a low rate of clinical response. $\begin{array}{llllll}4 & 5 & 7 & 9 & 21 & 22\end{array}$ Aggressive intradural manipulations, such as tonsillectomy, obex plugging and syringosubarachnoid shunt, do not guarantee improved efficacy, and serious consequences, such as respiratory distress syndrome, vascular injury, or even death, might still occur. ${ }^{2} 51723$ So far, it remains uncertain which procedure is the optimal surgical technique, and significant controversy surrounds the question of whether the dural opening and expansile duraplasty (PFDD vs DSD) are essential for adequate decompression. ${ }^{4} 7^{10}$ A randomised, controlled trial is needed to solve the clinical equipoise concerning the two surgical procedures. Hence we designed the trial to evaluate whether or not DSD is inferior to PFDD in the treatment of adult CM-I patients in terms of clinical improvement. We do not attempt to prove that DSD is superior to PFDD in terms of clinical improvement, because a small sample size fails to demonstrate any difference between the two surgical procedures.

In summary, DECMI is the first randomised controlled trial designed to evaluate whether DSD is an alternative treatment modality for CM-I patients, and whether it results in nearly equal efficacy, fewer complications, shorter length of operative time and hospital stay, better QoL and lower cost, in comparison with PFDD.

Contributors $\mathrm{SH}$ is the principle investigator and has led all stages of the study design. YH and JL drafted the manuscript. YH, JL, HC, JS, YF, QL, SG and YW participated in the design of the study and the protocol writing. All of the authors read and approved the final submitted manuscript.

Funding This work was supported by the science and technology support project of Sichuan Province (grant number 2012SZ0137).

Competing interests None declared.

Patient consent Obtained.

Ethics approval The study protocol was approved by the BMEC of West China Hospital on 7 January 2014. Substantial amendments of the study protocol must be approved by BMEC.

Provenance and peer review Not commissioned; externally peer reviewed.

Open Access This is an Open Access article distributed in accordance with the Creative Commons Attribution Non Commercial (CC BY-NC 4.0) license, which permits others to distribute, remix, adapt, build upon this work noncommercially, and license their derivative works on different terms, provided the original work is properly cited and the use is non-commercial. See: http:// creativecommons.org/licenses/by-nc/4.0/

\section{REFERENCES}

1. Hoffman CE, Souweidane MM. Cerebrospinal fluid-related complications with autologous duraplasty and arachnoid sparing in type I Chiari malformation. Neurosurgery 2008;62:156-60; discussion 160-1.

2. Klekamp J. Surgical treatment of Chiari I malformation-analysis of intraoperative findings, complications, and outcome for 371 foramen magnum decompressions. Neurosurgery 2012;71:365-80; discussion 380.

3. Godil SS, Parker SL, Zuckerman SL, et al. Accurately measuring outcomes after surgery for adult Chiari I malformation: determining the most valid and responsive instruments. Neurosurgery 2013;72:820-7; discussion 827.

4. Durham SR, Fjeld-Olenec K. Comparison of posterior fossa decompression with and without duraplasty for the surgical treatment of Chiari malformation type I in pediatric patients: a meta-analysis. $J$ Neurosurg Pediatr 2008;2:42-9.

5. Oro JJ, Mueller DM. Posterior fossa decompression and reconstruction in adolescents and adults with the Chiari I malformation. Neurol Res 2011;33:261-71.

6. Lee HS, Lee SH, Kim ES, et al. Surgical results of arachnoid-preserving posterior fossa decompression for Chiari I malformation with associated syringomyelia. $J$ Clin Neurosci 2012;19:557-60.

7. Litvack ZN, Lindsay RA, Selden NR. Dura splitting decompression for Chiari I malformation in pediatric patients: clinical outcomes, healthcare costs, and resource utilization. Neurosurgery 2013;72:922-9.

8. Tubbs RS, McGirt MJ, Oakes WJ. Surgical experience in 130 pediatric patients with Chiari I malformations. J Neurosurg 2003;99:291-6.

9. Chauvet D, Carpentier A, George B. Dura splitting decompression in Chiari type 1 malformation: clinical experience and radiological findings. Neurosurg Rev 2009;32:465-70.

10. Limonadi FM, Selden NR. Dura-splitting decompression of the craniocervical junction: reduced operative time, hospital stay, and cost with equivalent early outcome. J Neurosurg 2004;101(2 Suppl): $184-8$

11. Isu $\mathrm{T}$, Sasaki $\mathrm{H}$, Takamura $\mathrm{H}$, et al. Foramen magnum decompression with removal of the outer layer of the dura as treatment for syringomyelia occurring with Chiari I malformation. Neurosurgery 1993;33:844-9; discussion 849-50.

12. Gambardella G, Caruso G, Caffo M, et al. Transverse microincisions of the outer layer of the dura mater combined with foramen magnum decompression as treatment for syringomyelia with Chiari I malformation. Acta Neurochir (Wien) 1998;140:134-9.

13. Genitori L, Peretta P, Nurisso C, et al. Chiari type I anomalies in children and adolescents: minimally invasive management in a series of 53 cases. Childs Nerv Syst 2000;16:707-18.

14. Caldarelli M, Novegno F, Vassimi L, et al. The role of limited posterior fossa craniectomy in the surgical treatment of Chiar malformation Type I: experience with a pediatric series. J Neurosurg 2007;106:187-95

15. Hida K, Iwasaki Y, Koyanagi I, et al. Surgical indication and results of foramen magnum decompression versus syringosubarachnoid shunting for syringomyelia associated with Chiari I malformation. Neurosurgery 1995;37:673-8; discussion 678-9.

16. Munshi I, Frim D, Stine-Reyes R, et al. Effects of posterior fossa decompression with and without duraplasty on Chiari malformation-associated hydromyelia. Neurosurgery 2000;46:1384-9; discussion 1389-90.

17. Navarro R, Olavarria G, Seshadri R, et al. Surgical results of posterior fossa decompression for patients with Chiari I malformation. Childs Nerv Syst 2004:20:349-56.

18. Romero FR, Pereira CA. Suboccipital craniectomy with or without duraplasty: what is the best choice in patients with Chiari type 1 malformation? Arq Neuropsiquiatr 2010;68:623-6.

19. Hankinson T, Tubbs RS, Wellons JC. Duraplasty or not? An evidence-based review of the pediatric Chiari I malformation. Childs Nerv Syst 2011;27:35-40.

20. Mueller DM, Oro' JJ. The Chiari symptom profile: development and validation of a Chiari-/syringomyelia-specific questionnaire. J Neurosci Nurs 2013;45:205-10.

21. Ventureyra EC, Aziz HA, Vassilyadi M. The role of cine flow MRI in children with Chiari I malformation. Childs Nerv Syst 2003;19: 109-13.

22. Yeh DD, Koch B, Crone KR. Intraoperative ultrasonography used to determine the extent of surgery necessary during posterior fossa decompression in children with Chiari malformation type I. J Neurosurg 2006;105:26-32.

23. da Silva JA, dos Santos AA Jr, Melo LR, et al. Posterior fossa decompression with tonsillectomy in 104 cases of basilar impression, Chiari malformation and/or syringomyelia. Arq Neuropsiquiatr 2011;69:817-23. 